8. F. Ringleb, Beiträge zur Funktionentheorie in hyperkomplexen Systemen. I, Rend. Circ. Mat. Palermo vol. 57 (1933) pp. 311-340.

9. K. Shoda, Ein Kriterium für normale einfache hyperkomplexen Systeme, Proc. Imp. Acad. Japan, Tokyo vol. 10 (1934) pp. 195-197.

10. E. C. Titchmarsh, The theory of functions, Oxford University Press, 1947.

Duke UNIVERSITY AND

Case Institute of Technology

\title{
A GENERALIZATION OF HIRZEBRUCH POLYNOMIAL AND COBORDISM DECOMPOSITION
}

\section{YASURÔ TOMONAGA}

Introduction. In this paper we shall generalize the Hirzebruch polynomial and utilize it for the determination of cobordism components of a compact orientable and differentiable $4 k$-manifold.

1. Let $X^{4 k}$ be a compact orientable and differentiable $4 k$-manifold. According to Thom's theorem [1] such a manifold is "cobordant" with a polynomial of the complex projective spaces except torsion, i.e.

$$
X^{4 k} \approx \sum_{i_{1}+\cdots+i_{t}=k} A_{i_{1}+\cdots+i_{t}}^{k} P_{2 i_{1}}(c) \cdots P_{2 i_{t}}(c) \text { mod torsion }
$$

where $P_{i}(c)$ denotes the complex projective space of the complex dimension $i$ and $A$ 's denotes some rational numbers. The Hirzebruch polynomial is defined as follows [2]

$$
I_{i} \frac{\left(\gamma_{i}\right)^{1 / 2}}{\operatorname{tgh}\left(\gamma_{i}\right)^{1 / 2}}=\sum_{i=0}^{\infty} L_{i}\left(p_{1}, \cdots, p_{i}\right), \quad \sum_{i=0}^{\infty} p_{i}=I_{i}\left(1+\gamma_{i}\right)
$$

where $p_{i}$ denotes the Pontryagin class of dimension $4 i$. It is well known that

$$
L_{i}\left(p_{1}, \cdots, p_{i}\right)\left[P_{2 i}(c)\right]=1,
$$

from which we have

$$
L_{k}\left(p_{1}, \cdots, p_{k}\right)\left[X^{4 k}\right]=\text { index of } X^{4 k}=\sum_{i_{1}+\cdots+i_{t}=k} A_{i_{1} \cdots i_{t}}^{k} .
$$

We generalize (1.2) as follows:

\footnotetext{
Received by the editors September 9, 1960.
} 


$$
\begin{aligned}
& \prod_{i} \frac{\left(\gamma_{i}\right)^{1 / 2}}{\operatorname{tgh}\left(\gamma_{i}\right)^{1 / 2}}\left(1+y \operatorname{tgh}^{2}\left(\gamma_{i}\right)^{1 / 2}\right)=\sum_{i} \Gamma_{i}\left(y, p_{1}, \cdots, p_{i}\right) \\
& =1+\left(y+\frac{1}{3}\right) p_{1}+\left\{p_{2} y^{2}+\frac{1}{3}\left(4 p_{2}-p_{1}^{2}\right) y+\frac{1}{45}\left(7 p_{2}-p_{1}^{2}\right)\right\} \\
& \quad+\left\{p_{3} y^{3}+\frac{1}{3}\left(6 p_{3}-p_{1} p_{2}\right) y^{2}+\frac{1}{15}\left(17 p_{3}-8 p_{1} p_{2}+2 p_{1}^{3}\right) y\right. \\
& \left.\quad+\frac{1}{3^{3} \cdot 5 \cdot 7}\left(62 p_{3}-13 p_{1} p_{2}+2 p_{1}^{3}\right)\right\} \\
& \quad+\ldots
\end{aligned}
$$

and

$$
\begin{aligned}
& \prod_{i} \frac{\left(\gamma_{i}\right)^{1 / 2}}{\operatorname{tgh}\left(\gamma_{i}\right)^{1 / 2}}\left(1+y \operatorname{tgh}^{2}\left(\gamma_{i}\right)^{1 / 2}\right)^{-1}=\sum_{i} \Lambda_{i}\left(y, p_{1}, \cdots, p_{i}\right) \\
& =1+\left(\frac{1}{3}-y\right) p_{1} \\
& \quad+\left\{\left(p_{1}^{2}-p_{2}\right) y^{2}+\frac{1}{3}\left(p_{1}^{2}-4 p_{2}\right) y+\frac{1}{45}\left(7 p_{2}-p_{1}^{2}\right)\right\} \\
& \quad+\left\{-\left(p_{3}-2 p_{1} p_{2}+p_{1}^{3}\right) y^{3}+\left(-2 p_{3}+3 p_{1} p_{2}-p_{1}^{3}\right) y^{2}\right. \\
& \quad+\frac{1}{15}\left(-17 p_{3}+8 p_{1} p_{2}-2 p_{1}^{3}\right) y \\
& \left.\quad+\frac{1}{3^{3} \cdot 5 \cdot 7}\left(62 p_{3}-13 p_{1} p_{2}+2 p_{1}^{3}\right)\right\} \\
& +\cdots
\end{aligned}
$$

It follows from (1.4) and (1.5) that

(i) $\quad \Gamma_{i}\left(0, p_{1}, \cdots, p_{i}\right)\left[X^{4 i}\right]=$ index of $X^{4 i}$,

(ii) $\quad \Gamma_{i}\left(1, p_{1}, \cdots, p_{i}\right)\left[X^{4 i}\right]=2^{2 i}\left(\right.$ index of $\left.X^{4 i}\right)$,

(iii) $\Gamma_{i}\left(-1, p_{1}, \cdots, p_{i}\right)\left[X^{4 i}\right]=A$-genus of $X^{4 i}[2$, p. 14].

Moreover we can prove that $\Gamma_{i}\left(y, p_{1}, \cdots, p_{i}\right)\left[X^{4 i}\right]$ has integral coefficients. The complete proof will be given in another paper but the proof is easy in the case of an almost complex split manifold because $\Gamma_{i}\left(X^{4 i}\right)$ decomposes into many virtual indices [2, p. 87].

2. Next we consider the application of our multiplicative series for the determination of cobordism coefficients. We have from (1.1) 


$$
\begin{aligned}
& \Lambda_{k}\left(y, p_{1}, \cdots, p_{k}\right)\left[X^{4 k}\right] \\
& \quad=\sum_{i_{1}+\cdots+i_{t}=k} A_{i_{1} \cdots i_{t}}^{k} \Lambda_{i_{1}}\left[P_{2 i_{1}}(c)\right] \cdots \Lambda_{i_{t}}\left[P_{2 i_{t}}(c)\right] \quad(k \leqq 4) .{ }^{1}
\end{aligned}
$$

Comparing the coefficients of $y^{a}$ s $(a=0, \cdots, k)$ we have

$$
\begin{aligned}
& A_{2}^{2}=\frac{1}{5}\left(-2 p_{2}+p_{1}^{2}\right)\left[X^{8}\right], \quad A_{11}^{2}=\frac{1}{9}\left(5 p_{2}-2 p_{1}^{2}\right)\left[X^{8}\right], \\
& A_{3}^{3}=\frac{1}{7}\left(3 p_{3}-3 p_{1} p_{2}+p_{1}^{3}\right)\left[X^{12}\right],
\end{aligned}
$$

$$
\begin{aligned}
A_{21}^{3} & =\frac{1}{15}\left(-21 p_{3}+19 p_{1} p_{2}-6 p_{1}^{3}\right)\left[X^{12}\right], \\
A_{111}^{3} & =\frac{1}{27}\left(28 p_{3}-23 p_{1} p_{2}+7 p_{1}^{3}\right)\left[X^{12}\right] \\
A_{4}^{4} & =\frac{1}{9}\left(-4 p_{4}+4 p_{1} p_{3}+2 p_{2}^{2}-4 p_{1}^{2} p_{2}+p_{1}^{4}\right)\left[X^{16}\right], \\
A_{31}^{4} & =\frac{1}{21}\left(36 p_{4}-33 p_{1} p_{3}-18 p_{2}^{2}+33 p_{1}^{2} p_{2}-8 p_{1}^{4}\right)\left[X^{16}\right],
\end{aligned}
$$

$$
A_{22}^{4}=\frac{1}{25}\left(18 p_{4}-18 p_{1} p_{3}-7 p_{2}^{2}+16 p_{1}^{2} p_{2}-4 p_{1}^{4}\right)\left[X^{16}\right]
$$

$$
\begin{aligned}
A_{211}^{4} & =\frac{1}{45}\left(-180 p_{4}+159 p_{1} p_{3}+80 p_{2}^{2}-150 p_{1}^{2} p_{2}+36 p_{1}^{4}\right)\left[X^{16}\right] \\
A_{1111}^{4} & =\frac{1}{81}\left(165 p_{4}-137 p_{1} p_{3}-70 p_{2}^{2}+127 p_{1}^{2} p_{2}-30 p_{1}^{4}\right)\left[X^{16}\right] .
\end{aligned}
$$

3. Next we consider the case where a $X^{4 k}$ is a submanifold of $X^{4 k+2 r}$ where we assume that both manifolds be compact orientable and differentiable. Let $X^{4 k}$ be determined by the cohomology classes $v_{1}, \cdots, v_{r} \in H^{2}\left(X^{4 k+2 r}, Z\right)$. Then we can determine the cobordism coefficients of $X^{4 k}$ by $v^{\prime}$ s and the Pontryagin classes of $X^{4 k+2 r}$ as follows:

$X^{8} \subset X^{10}$

$$
\begin{aligned}
& A_{2}^{2}=\frac{1}{5}\left(-v^{5}-2 v p_{2}+v p_{1}^{2}\right)\left[X^{10}\right], \\
& A_{11}^{2}=\frac{1}{9}\left(3 v^{5}-v^{3} p_{1}+5 v p_{2}-2 v p_{1}^{2}\right)\left[X^{10}\right],
\end{aligned}
$$

\footnotetext{
${ }^{1} \Gamma_{k}$ is also available for this purpose but for $k \leqq 3$.
} 
$X^{12} \subset X^{14}$

$$
\begin{aligned}
\dot{A}_{3}^{3}= & \frac{1}{7}\left\{-v^{7}+\left(3 p_{3}-3 p_{1} p_{2}+p_{1}^{3}\right) v\right\}\left[X^{14}\right], \\
A_{21}^{3}=\frac{1}{15}\left\{8 v^{7}-v^{5} p_{1}+v^{3}\left(2 p_{2}-p_{1}^{2}\right)\right. & \left.+v\left(-21 p_{3}+19 p_{1} p_{2}-6 p_{1}^{3}\right)\right\}\left[X^{14}\right], \\
A_{111}^{3}=\frac{1}{27}\left\{-12 v^{7}+3 v^{5} p_{1}\right. & +\left(2 p_{1}^{2}-5 p_{2}\right) v^{3} \\
& \left.+\left(28 p_{3}-23 p_{1} p_{2}+7 p_{1}^{3}\right) v\right\}\left[X^{14}\right],
\end{aligned}
$$

$X^{8} \subset X^{12}$

$$
\begin{aligned}
A_{2}^{2}= & \left\{-\frac{1}{5}\left(v_{1}^{5} v_{2}+v_{2}^{5} v_{1}\right)+\frac{1}{5}\left(p_{1}^{2}-2 p_{2}\right) v_{1} v_{2}\right\}\left[X^{12}\right], \\
A_{11}^{2}=\left\{\frac{1}{3}\left(v_{1}^{5} v_{2}+v_{2}^{5} v_{1}\right)+\frac{1}{9} v_{1}^{3} v_{2}^{3}-\right. & \frac{1}{9}\left(v_{1} v_{2}^{3}+v_{2} v_{1}^{3}\right) p_{1} \\
& \left.+\frac{1}{9}\left(5 p_{2}-2 p_{1}^{2}\right) v_{1} v_{2}\right\}\left[X^{12}\right] .
\end{aligned}
$$

The method used here was as follows [2, p. 87]: From (2.1) replaced by $\Gamma_{k}$ and the relation

$$
\begin{aligned}
\Gamma_{k}\left(y, p_{1}, \cdots, p_{k}\right)\left[X^{4 k}\right] & \\
=\left[\kappa ^ { 4 k + 2 r } \left\{\left(\frac{\operatorname{tgh} v_{1}}{1+y \operatorname{tgh}^{2} v_{1}}\right)\right.\right. & \cdots\left(\frac{\operatorname{tgh} v_{r}}{1+y \operatorname{tgh}^{2} v_{r}}\right) \\
& \left.\left.\cdot \sum_{i} \Gamma_{i}\left(y, p_{1}, \cdots, p_{i}\right)\right\}\right]\left[X^{4 k+2 r]}\right.
\end{aligned}
$$

we obtain (3.1)-(3.3) by comparing the coefficients of $y^{a,}$ s.

\section{REFERENCES}

1. R. Thom, Quelques proprietés globales des varietés differentielles, Comment. Math. Helv. vol. 28 (1954) pp. 17-86.

2 F. Hirzebruch, Neue topologische Methoden in der algebraischen Geometrie, Berlin, Springer, 1956.

UTSUNOMIYA UNIVERSITY, JAPAN 